\title{
Study Subject Protocol Version Relationship Effective Date Range
}

National Cancer Institute

\section{Source}

National Cancer Institute. Study Subject Protocol Version Relationship Effective Date

Range. NCI Thesaurus. Code C94156.

The date and time span specifying when the relationship between a study subject and protocol version begins and ends. 